Stichprobeninventur in der Praxis

Zielgruppe: Qualifizierte Mitarbeiter aus Rechnungswesen, Revision, Lagerwirtschaft, Organisation und EDV-Anwendungsberatung sowie der Wirtschaftsprüfung.

Referenten: K.-P. Schuster, Philips GmbH, Hamburg Prof. Dr. V. Steinecke, HOESCH Werke AG, Dortmund

Termine u. 13./14. Februar 1984 in Köln

Orte: $\quad 19 . / 20$. November 1984 in Hamburg

Gebühren: DM 990,- zuzügl. MWST.
Prognoseverfahren in der Praxis

Zielgruppe: Qualifizierte Mitarbeiter aus Marketing/Marktforschung und -planung, Material- und Lagerwirtschaft

Referent: Prof. Dr. J. Schwarze, TU Braunschweig

Termine u. 10./11. Mai 1984 in Dortmund

Orte: $\quad$ 10./11. September 1984 in München

Gebühren: DM 890,- zuzügl. MWST.

Auskünfte zu den mbp-Seminaren erteilt:

Mathematischer Beratungs- und Programmierdienst GmbH

z. H. Frau Bleeker, Semerteichstraße 47, 4600 Dortmund 1

\title{
Eingegangene Bücher
}

Die im folgenden aufgelisteten Bücher sind zur Besprechung im OPEERATIONS RESEARCH-SPEKTRUM eingegangen.

Besprechungstexte zu einzelnen dieser Bücher werden in den folgenden Heften erscheinen.

Becker, M., Ebner, M.: Planen und Entscheiden mit Operations Research. Zürich: Verlag Industrielle Organisation 1983.3. Auflage, 141 Seiten, Fr. 24,--

Berndt, R.: Einführungsplanungen neuer Produkte bei unsicheren Erwartungen. Göttingen, Zürich: Vandenhoeck \& Ruprecht 1983. XIV, 384 Seiten, DM 85,-.

Bogardi, L., David, L., Duckstein L.: Trade-off Between Cost and Effectiveness of Control of Nutrient Loading into a Water Body. Laxenburg: IIASA 1983. 36 pages, \$ 6.00.

Gould, F. J., Tolle, J. W.: Complementary Pivoting on a Pseudomanifold Structure with Applications in the Decision Sciences. Berlin: Heldermann 1983. IV, 204 pages, DM 58.-.

Haustein, H.D., Maier, H., Ulrich, E.: Innovation and Requirements for Human Resources. Laxenburg: IIASA 1983. 44 pages, $\$ 6.00$.

Jansen, J., Marcotorchino, J. F., Proth, J. M. (Eds.): New trends in data analysis and applications. Amsterdam, New York, Oxford: North-Holland 1983. XI, 273 pages, hIl. 120.00, US-\$ 51.00 .
Kim, .K. H., Roush, F. W.: Competitive economics: Equilibrium and Arbitration. Amsterdam, New York, Oxford: NorthHolland 1983. XV, 283 pages, hfl. 150.00.

Mirman, L. J., Spulber, D. F. (Eds.): Essays in the Economics of Renewable Resources. Amsterdam, New York, Oxford: NorthHolland 1982. IX, 287 pages, hfl. 140.00.

Nahorski, Z., Ravn, H. F., Vidal, R. V. V.: Optimization of Discrete Time Systems. Lecture Notes in Control and Information Sciences, Vol. 51. Berlin, Heidelberg, New York, Tokyo: Springer 1983. 137 Seiten, DM 19,50.

Piehler, J.: Algebraische Methoden in der ganzzahligen Optimierung. Teubner-Texte, Band 51. Leipzig: B. G. Teubner 1983. 99 Seiten, DM 11,-.

Steffen, R.: Produktions- und Kostentheorie. Stuttgart, Berlin, Köln, Mainz: W. Kohlhammer 1983.136 Seiten, DM 24,--.

Troßmann, E.: Verschnittoptimierung, dargestellt an Beispielen aus der Textilindustrie. Berlin: Duncker \& Humblot 1983. 213 Seiten, DM 78,-.

Uronen, P.: Integrated Computer Systems in the Pulp and Paper Industry. Laxenburg: IIASA 1983. 36 pages, \$ 6.00.

\section{Buchbesprechungen}

Malliaris, A.G., Brock, W.A.: Stochastic in Economics and Finance. Advanced Textbooks in Economics, Vol. 17. Amsterdam, New York, Oxford: North-Holland 1982. XVI, 278 pages, US-\$ 35,-, hfl. 85,--.

Das vorliegende Textbuch ist als einführende Übersicht über fortgeschrittene stochastische Methoden und deren Anwendung in der Wirtschafts- und Finanzierungstheorie gedacht, die als Anleitung für die Lektüre weiterführender Literatur dienen soll.

$\mathrm{Zu}$ diesem $\mathrm{Zweck}$ werden in einem ersten Teil des Buches die methodischen Grundlagen gelegt: Das erste Kapitel (S. 1-63) gibt einen knappen Überblick über die moderne Wahrscheinlichkeitstheorie. Während die Ausführungen über die Grundlagen der Wahrscheinlichkeitstheorie relativ knapp gehalten sind, werden Martingale und deren Anwendungen, Stochastische Prozesse und optimale Stopp-Probleme ausfïhrlich angesprochen. Das zweite Kapitel (S. 65-140) befaßst sich mit der stochastischen Infinitesimalrechnung. Ausgehend von einfachen Beispielen für dy- namische stochastische Modelle werden stochastische Differential- und Integralgleichungen eingeführt. Im Mittelpunkt dieses Kapitels steht das Lemma von Itô sowie die Eigenschaften stochastischer Differentialgleichungen vom Itô-Typ. Abschließend werden einige Grundlagen der stochastischen Kontrolltheorie behandelt.

Der zweite Teil des Buches ist der Anwendung dieser Ansätze auf stochastische, dynamische Probleme der Wirtschaftswissenschaften gewidmet. Im dritten Kapitel (S. 141-216) werden Anwendungen in der Wirtschaftstheorie untersucht, insbesondere werden Probleme der Wachstumstheorie sowie der Investitionsund Kapitaltheorie, aber auch der Inflationstheorie unter stochastischen Bedingungen angesprochen. Das abschließende vierte Kapitel (S. 217-278) befaßt sich mit Anwendungen in der Finanzierungstheorie; es werden insbesondere das Black-Scholes-Modell für Optionspreise, die dynamische Portfolio-Theorie und das Capital-Asset-Pricing-Modell sowie die Klassenhaltungstheorie angesprochen. 